\title{
A continuous process for glyoxal valorisation using tailored Lewis- acid zeolite catalysts
}

\section{Journal Article}

\section{Author(s):}

Dapsens, Pierre Y.; Mondelli, Cecilia; Kusema, Bright T.; Verel, René (D); Pérez-Ramírez, Javier

Publication date:

2014-03-01

Permanent link:

https://doi.org/10.3929/ethz-a-010792728

Rights / license:

In Copyright - Non-Commercial Use Permitted

Originally published in:

Green Chemistry 16(3), https://doi.org/10.1039/c3gc42353k

Funding acknowledgement:

140496 - Biomass to chemicals over tailored hierarchical zeolite-based catalysts (SNF) 


\title{
Continuous Process for Glyoxal Valorisation using Tailored Lewis-acid Zeolite Catalysts
}

\author{
Pierre Y. Dapsens, Cecilia Mondelli*, Bright T. Kusema, René Verel and Javier Pérez-Ramírez* \\ Received (in $X X X, X X X)$ Xth $X X X X X X X X X 20 X X$, Accepted Xth XXXXXXXXX 20XX \\ ${ }_{5}$ DOI: 10.1039/b000000x
}

The aqueous-phase heterogeneously catalysed isomerisation of bio-oil derived glyoxal is herein introduced as a novel route for the sustainable production of glycolic acid. While commercial ultra-stable Y zeolites displayed only moderate performance, their evaluation enabled to highlight the crucial role of Lewis acidity for the reaction. Gallium incorporation in these zeolites boosted the glycolic acid yield, but 10 the best catalytic results were obtained over tin-containing MFI-type zeolites, reaching $91 \%$ yield of the desired product at full conversion. These materials comprised hydrothermally-synthesised Sn-MFI as well as a novel catalyst obtained by the introduction of tin in silicalite- 1 by means of a simpler and more scalable method, i.e. alkaline-assisted metallation. In-depth spectroscopic characterisation of these systems uncovered a substantial similarity of the tin centres obtained by the top-down and bottom-up 15 synthetic approaches. NMR spectroscopic studies gave evidence that the reaction follows a 1,2-hydride shift mechanism solely catalysed by Lewis-acid sites. The Sn-MFI analogue could be reused in 5 cycles without the need for intermediate calcination, did not evidence any tin leaching, and demonstrated suitable for utilisation under continuous-flow operation. Both tin-based zeolites exhibited remarkable performance also in alcoholic solvents, leading to the one-pot production of relevant alkyl glycolates.

\section{${ }_{20} 1$ Introduction}

Nowadays, chemical companies are increasingly investing on the manufacture of polymers derived from renewable resources (socalled biopolymers), in view of reducing their carbon footprint as well as of expanding their products' portfolio.[1] In this context, ${ }_{25} \alpha$-hydroxy acids (AHA) such as lactic acid (LA, $\mathrm{C}_{3}$ molecule) and glycolic acid (GA, $\mathrm{C}_{2}$ molecule) comprise monomers of primary relevance. Besides for the plastics obtained from the individual AHAs, that is, polylactide (PLA) and polyglycolide (PGA), a mixed polymer, poly(lactic-co-glycolic) acid (PLGA), 30 can be derived. PLA constitutes a novel functional material mainly used in the packaging industry, while PGA and PLGA are extensively employed in the medical field owing to their biocompatibility and exceptional biodegradability.[2] Significant research efforts have been devoted to the preparation of bio-based 35 LA.[3] In contrast, the production of GA from bio-derived feedstocks has been investigated to a limited extent. Still, its demand reached $40 \mathrm{ktons}$ in 2011 and is expected to double by 2018 , considering an annual growth rate estimated at $10.1 \%$.[4]

GA is currently manufactured by the carbonylation of ${ }_{40}$ formaldehyde in the presence of $\mathrm{H}_{2} \mathrm{SO}_{4}$. Disadvantageously, this process requires elevated temperatures and pressures $(473 \mathrm{~K}$, 300 bar) to attain appreciable yields of GA. Nonetheless, significant amounts of formic acid by-product and unreacted formaldehyde are still contained in the final mixture.[5] Other 45 processes for GA production have been reported such as the saponification of chloroacetic acid, the enzymatic conversion of glycolonitrile, and the electrolytic reduction of oxalic acid.[6] These routes are impractical for industrial scale due to energy intensiveness, environmental issues, long reaction times, and 50 scarce selectivity. Thus, based on the present scenario, the availability of an efficient, affordable, and scalable chemocatalytic route to transform biomass into glycolic acid under mild conditions would be highly valuable. A few attempts have been made in this direction. Zhang et al. have recently 55 investigated the direct conversion of cellulose into GA over Mocontaining heteropolyacids at $453 \mathrm{~K}$ and 6 bar $\mathrm{O}_{2}$, reaching a maximum yield of $49 \%$ at $91 \%$ conversion.[7] Besides for the limited selectivity of this catalytic system, the use of heteropolyacids remains problematic separation-wise. An 60 alternative route to GA encompasses the oxidation of ethylene glycol, which can be produced from xylan hemicellulose by hydrolysis to xylose, hydrogenation of the sugar to xylitol, and hydrogenolysis of the polyol.[8] Drawbacks of this approach are the multiplicity of steps, the requirement for expensive metals ${ }_{65}$ such as $\mathrm{Ru}$ and $\mathrm{Pt}$ in their majority, and the low selectivity ( $c a$. $33 \%$ at any conversion level) to ethylene glycol in the hydrogenolysis step. Another possible pathway is represented by the oxidation of glycerol. Still, at present this chemocatalytic process yields a maximum of $17 \%$ GA over $\mathrm{Nb}_{2} \mathrm{O}_{5}$ and $40 \%$ GA 70 and $60 \%$ glycolate over Au-based catalysts.[9]

Herein, we put forward a novel synthesis path for GA, which only comprises the heterogeneously catalysed isomerisation of glyoxal (GLY) (Scheme 1) in water. In addition to the low 


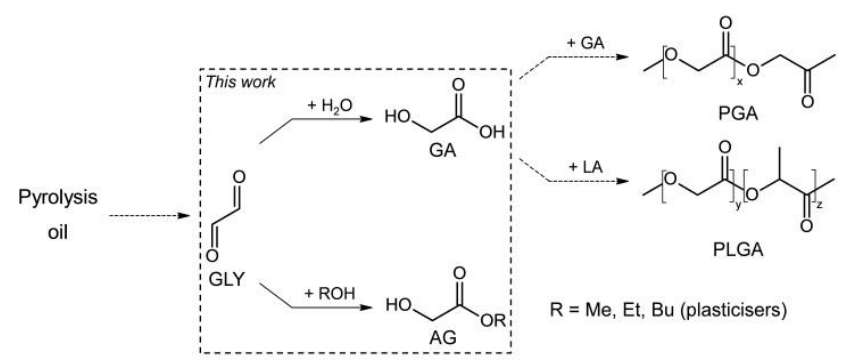

Scheme 1 Strategy for the production of glycolic acid and alkyl glycolates based on the Lewis-acid catalysed isomerisation of glyoxal.

temperature $(363 \mathrm{~K})$ and pressure (ambient) required, our interest in this reaction, demonstrated years back at the laboratory level 5 with homogeneous bases or acids, [10] derived from the fact that GLY represents an undesired oxygenated molecule contained in bio-oil.[11] The occurrence of this compound is indicated to be independent of the nature of the lignocellulosic feedstock that undergoes the pyrolytic treatment and to correspond to $c a$. 2$103 \mathrm{wt} . \%$ of the obtained liquid mixture.[12] As exemplified by the case of Finland, where installations with an annual production capacity of pyrolysis oil of $50 \mathrm{ktons} / \mathrm{year}$ are being erected, a significant amount of glyoxal is expected to be available in the coming years.[13] Furthermore, GLY recovery from this 15 renewable source can be easily accomplished by water extraction followed by chromatographic purification.[14] Thus, the isomerisation of GLY to GA appears as an attractive route to valorise a waste product along the biofuels manufacture chain. This approach follows the biorefinery concept, which is one way 20 how to render biofuels production more economical and sustainable in the long term, i.e. through the extra-profit and higher efficiency provided by the co-generation of chemicals. Last but not least, since GA does not possess chiral centres, there is no obvious drawback in using a solid catalyst. This contrasts 25 the production of LA, where the desired L-isomer cannot be so far obtained selectively over a heterogeneous system.[15]

Recent DFT studies by Oshima et al. have indicated that a mechanism based on a Lewis-acid catalysed 1,2-hydride shift is energetically more favourable than a pathway relying on a 30 Brønsted-acid catalysed dehydration-tautomerisation for the isomerisation of GLY to GA.[16] This prediction is in analogy with the reaction scheme for the aldo-ketose isomerisation of sugars and with the conversion of pyruvaldehyde into LA.[17] Tin-containing porous materials (e.g. Sn-BEA, Sn-MWW, Sn35 MCM-41, and Sn-CSM) exhibit excellent performances in the latter reactions, greatly surpassing commercial aluminosilicate zeolites. Their remarkable activity and selectivity are mainly associated with the sole presence of strong and well-defined Lewis-acid centres enabling high conversion rates with minimal 40 by-products formation.[17,18] Alternatively, post-synthetic modification of commercial zeolites has been uncovered as a suitable tool to create tin-free zeolites with tailored properties. In particular, the alumination of MFI-type zeolites and galliation of USY zeolites in alkaline media have enabled to introduce unique 45 tetra-coordinated Lewis-acid $\mathrm{Al}$ and $\mathrm{Ga}$ sites in the zeolitic frameworks, which have demonstrated highly selective for the production of LA and its esters derivatives.[19]

Based on these premises, the aqueous-phase isomerisation of glyoxal to glycolic acid was initially studied over commercially
50 available USY zeolites to discriminate the role of the nature of acidity. Then, state-of-the-art Lewis-acid catalysts were evaluated, which indicated the superiority of hydrothermallysynthesised Sn-MFI with respect to gallium-based systems. Following this result, a novel catalyst was designed by 55 incorporation of tin in silicalite- 1 through a simpler and more scalable method, i.e. alkaline treatment in the presence of a soluble tin salt. The characteristics of the tin sites were in-depth investigated by spectroscopic methods in terms of acidity and structure to explain the similar catalytic results obtained over the ${ }_{60}$ two differently prepared catalysts. NMR spectroscopy was applied to provide experimental support to the postulated 1,2hydride shift route for GLY isomerisation. Industrially relevant aspects of the reaction over the modified silicalite- 1 catalyst were evaluated, such as catalyst recyclability, tin leaching, use of ${ }_{65}$ higher feed concentrations, and the possibility of a continuousflow operation. Finally, it was explored whether the one-pot production of prominent alkyl glycolates, applied as plasticisers and chemical intermediates,[20] would be possible over the tincontaining zeolites when using an alcohol as reaction medium 70 instead of water.

\section{Experimental}

\subsection{Catalyst synthesis}

USY zeolites (CBV300, CBV500, CBV600, CBV712, CBV720, ${ }_{75}$ CBV760, and CBV780) were purchased from Zeolyst International, whereas high-silica USY (HSZ390 HUA) and silicalite-1 (HSZ890 H0A) were supplied by Tosoh Corporation. Prior to their utilisation, the as-received zeolites were converted into their protonic form by calcination in static air at $823 \mathrm{~K}$ $80\left(5 \mathrm{~K} \mathrm{~min}^{-1}\right)$ for $5 \mathrm{~h}$. The composition and textural properties of these samples are reported in Table SI1. Reference catalysts comprised Amberlyst-15 (Dow Chemical Company) and $\mathrm{SnO}_{2}$ (Sigma-Aldrich, 99.9\%).

Ti-MFI was synthesised following the protocol reported by ${ }_{85}$ Huang et al. [21] Thus, a mixture containing TEOS $\left(24.5 \mathrm{~cm}^{3}\right.$, Sigma-Aldrich, 98\%), TPAOH (17.6 cm $\mathrm{cm}^{3}$, Alfa Aesar, 35 wt.\%), and deionised $\mathrm{H}_{2} \mathrm{O}\left(28.3 \mathrm{~cm}^{3}\right)$ was allowed to react under stirring at room temperature for $1 \mathrm{~h}$. Thereafter, the solution was cooled down to $273 \mathrm{~K}$ in an ice bath and a solution of TBOT $\left(1.2 \mathrm{~cm}^{3}\right.$, ${ }_{90}$ TCI, 98\%) in EtOH (12.5 $\mathrm{cm}^{3}$, Scharlau, 99.9\%) was added dropwise under vigorous stirring. After further stirring for $1 \mathrm{~h}$ at the same temperature, the mixture was heated to $333 \mathrm{~K}$ for $1 \mathrm{~h}$ to enable the hydrolysis of TBOT. This resulted in the formation of a sol with a molar composition of $\mathrm{SiO}_{2}: 0.033 \mathrm{TiO}_{2}$ : 950.2 TPAOH : 2 ethanol : $20 \mathrm{H}_{2} \mathrm{O}$. The sol was transferred into a $100-\mathrm{cm}^{3}$ Teflon-lined autoclave, which was placed in an oven and heated at $443 \mathrm{~K}$ for 4 days under static conditions. The obtained solid was recovered by filtration, washed thoroughly with deionised water, dried overnight at $338 \mathrm{~K}$, and finally calcined in 100 static air at $823 \mathrm{~K}\left(5 \mathrm{~K} \mathrm{~min}^{-1}\right)$ for $5 \mathrm{~h}$ to ensure the complete removal of the organic pore directing agent.

Sn-MFI was synthesised according to the method developed by Mal et al. [22] TEOS (93.7 g, Sigma-Aldrich, 98\%) was mixed with a solution of $\mathrm{SnCl}_{4} \cdot 5 \mathrm{H}_{2} \mathrm{O}(1.26 \mathrm{~g}$, Sigma-Aldrich, $\left.{ }_{105} 98 \%\right)$ in deionised $\mathrm{H}_{2} \mathrm{O}(30 \mathrm{~g})$ and allowed to react for $30 \mathrm{~min}$ 
under stirring. Then, TPAOH (198 g, Alfa Aesar, 20 wt.\%) was added drop-wise and the mixture was stirred for another hour. Finally, $84 \mathrm{~g}$ of deionised $\mathrm{H}_{2} \mathrm{O}$ were added and the solution was stirred for $30 \mathrm{~min}$. The final molar ratio of the synthesis gel was ${ }_{5} 0.008 \mathrm{SnO}_{2}: 1 \mathrm{SiO}_{2}: 0.44 \mathrm{TPAOH}: 34.3 \mathrm{H}_{2} \mathrm{O}$. The sol was transferred into a $500-\mathrm{cm}^{3}$ Teflon-lined autoclave, which was heated in an oven at $433 \mathrm{~K}$ for 3 days under static conditions. The resulting crystals were separated by filtration and subjected to the same work up as described for Ti-MFI.

10 CBV720 and HSZ890 H0A (hereafter denoted as MFI), referred to as parent (code $\mathrm{P}$ ), were used for post-synthetic modification, that is alkaline-assisted metallation. The latter consisted in an alkaline treatment in an aqueous $0.3 \mathrm{M} \mathrm{NaOH}$ solution $\left(30 \mathrm{~cm}^{3}\right.$ per gram of dried zeolite), containing either $150.05 \mathrm{M}$ of $\mathrm{Ga}\left(\mathrm{NO}_{3}\right)_{3}$ (Sigma-Aldrich, $99.9 \%$ ) or of $\mathrm{Sn}\left(\mathrm{SO}_{4}\right)_{2}$ (Acros, $98 \%$ ) at $338 \mathrm{~K}$ for $30 \mathrm{~min}$ in an Easymax ${ }^{\mathrm{TM}} 102$ reactor (Mettler Toledo). In the case of the alkaline solution containing tin sulfate, owing to the rather low solubility of the tin hydroxide formed, the mixture was heated at $318 \mathrm{~K}$ for $1 \mathrm{~h}$ and filtered to 20 remove the precipitate prior to the addition of the zeolite. The resulting modified metallosilicates were converted into their protonic form by three consecutive ion exchanges in an aqueous solution of ammonium nitrate $\left(0.1 \mathrm{M} \mathrm{NH} \mathrm{NNO}_{3}, 6 \mathrm{~h}, 298 \mathrm{~K}\right.$, $100 \mathrm{~cm}^{3}$ per gram of dried zeolite) followed by calcination in 25 static air at $823 \mathrm{~K}\left(5 \mathrm{~K} \mathrm{~min}^{-1}\right)$ for $5 \mathrm{~h}$. These samples are denoted by the codes CBV720-ATGa and MFI-ATSn, respectively.

\subsection{Characterisation}

The content of $\mathrm{Si}, \mathrm{Al}, \mathrm{Ga}$, and $\mathrm{Sn}$ in the catalysts and in the 30 filtrate obtained after catalyst preparation was determined by inductively coupled plasma optical emission spectroscopy (ICPOES) using a Horiba Ultra 2 instrument equipped with a photomultiplier tube detector. Prior to the measurements, the materials were digested in an acidic mixture $\left(\mathrm{HCl} / \mathrm{HNO}_{3} / \mathrm{HF}\right)$ 35 under heating ( $343 \mathrm{~K}$, overnight). Nitrogen sorption at $77 \mathrm{~K}$ was performed using a Quantachrome Quadrasorb-SI analyser on degassed samples $\left(10^{-1} \mathrm{mbar}, 573 \mathrm{~K}, 3 \mathrm{~h}\right)$. Powder X-ray diffraction (XRD) was measured using a PANalytical X'Pert PRO-MPD diffractometer with Ni-filtered $\mathrm{Cu} \mathrm{K} \alpha$ radiation ${ }_{40}(\lambda=0.1541 \mathrm{~nm})$. Data was recorded in the $5-70^{\circ} 2 \theta$ range with an angular step size of $0.05^{\circ}$ and a counting time of $7 \mathrm{~s}$ per step. Transmission electron microscopy (TEM) was undertaken using a FEI Tecnai F30 microscope operated at $300 \mathrm{kV}$ (field emission gun). The samples were prepared by depositing a few droplets of 45 zeolite suspension in methanol onto a carbon-coated copper grid, followed by evaporation at room temperature. Fourier transform infrared (FTIR) spectroscopy measurements of adsorbed pyridine/d3-acetonitrile were carried out using a Bruker IFS66 spectrometer equipped with a liquid $\mathrm{N}_{2}$-cooled mercury cadmium 50 telluride (MCT) detector. Self-supporting zeolite wafers $(20 \mathrm{mg}$, 5 ton $\mathrm{cm}^{-2}, 1 \mathrm{~cm}^{2}$ ) were pretreated at $10^{-3}$ mbar and $693 \mathrm{~K}$ for $4 \mathrm{~h}$. After cooling down to room temperature, the sample was saturated with pyridine/d3-acetonitrile vapour and then evacuated at room temperature (15 $\mathrm{min})$. In the case of pyridine, subsequent 55 desorption steps at $473 \mathrm{~K}(30 \mathrm{~min}), 523 \mathrm{~K}(20 \mathrm{~min}), 573 \mathrm{~K}$ ( $20 \mathrm{~min})$, and $623 \mathrm{~K}$ (20 min) were performed. Spectra were recorded in the $650-4000 \mathrm{~cm}^{-1}$ range with a resolution of $4 \mathrm{~cm}^{-1}$ by co-addition of 32 scans. UV-Vis spectroscopy was performed with an Ocean Optics UV-Vis spectrophotometer in diffuse 60 reflectance mode using barium sulfate for the background collection. Prior to the analysis, the samples were dried at $573 \mathrm{~K}$ for $3 \mathrm{~h}$ under vacuum $\left(10^{-1} \mathrm{mbar}\right)$. X-ray photoelectron spectroscopy (XPS) was carried out under UHV $\left(10^{-8} \mathrm{mbar}\right)$ using a VG Thermo Escalab 220i-XL spectrometer equipped with 65 a high intensity twin-anode ( $\mathrm{Al} \mathrm{K} \alpha$ radiation) source. The spectra were charge-corrected with respect to the $\mathrm{C} 1 s$ signal at $284.5 \mathrm{eV}$.

\subsection{NMR spectroscopy}

${ }^{13} \mathrm{C}$ and DEPT-135 [23] nuclear magnetic resonance (NMR) spectroscopic studies were applied to unravel the mechanism of ${ }_{70}$ GLY isomerisation to GA. Measurements were carried out in a Bruker Avance III HD spectrometer (Bruker Biospin) operating at a ${ }^{1} \mathrm{H}$ Larmor frequency of $500.13 \mathrm{MHz}\left(125 \mathrm{MHz}\right.$ for $\left.{ }^{13} \mathrm{C}\right)$, using a $\mathrm{BBFO}$ probe. The ${ }^{13} \mathrm{C}$ experiment was a standard broadband ${ }^{1} \mathrm{H}$ decoupled sequence using a WALTZ-16 [24], in 75 which 128 scans were coadded. For the DEPT spectrum, 2048 scans were coadded using the standard pulse sequence (also with WALTZ-16 ${ }^{1} \mathrm{H}$ decoupling) with a recycle delay of $2 \mathrm{~s}$. Both experiments used a spectral width of 236.5 ppm centered at 100 ppm and consisted of 65000 points. The phase of the DEPT 80 spectrum was adjusted using the $\mathrm{CH}_{3}$ signal of TSP-d4, which was added to the samples to serve as reference. The spectra were processed using the Bruker Topspin 3.2 software.

\subsection{Catalytic tests}

${ }_{85}$ Batch catalytic tests were performed under autogenous pressure in $15-\mathrm{cm}^{3}$ thick-walled glass vials (Ace, pressure tubes, front seal) dipped in an oil bath heated at the desired reaction temperature ( 363 or $373 \mathrm{~K}$ ). The vials were loaded with $200 \mathrm{mg}$ of glyoxal (GLY, Sigma-Aldrich, 95\%, trimer), $80 \mathrm{mg}$ of catalyst, 90 and $3.80 \mathrm{~g}$ of solvent. The latter comprised water, deuterated water $\left(\mathrm{D}_{2} \mathrm{O}\right.$, Sigma-Aldrich, $100 \%$, for the NMR spectroscopic studies), methanol, ethanol (both purchased from Scharlau, 99.9\%), or butan-1-ol (Merck, 99.5\%). The mixture was allowed to react under vigorous stirring during 0.5 to $48 \mathrm{~h}$. Then, the 95 reaction was quenched using an ice bath and the catalyst removed by means of a Chromafil Xtra $0.25 \mu \mathrm{m}$ syringe filter. Continuousflow catalytic tests were conducted in a $\mathrm{H}$-Cube $\mathrm{Pro}^{\mathrm{TM}}$ reactor system (Thales Nanotechnology) equipped with a fixed-bed micro-reactor (Catalyst cartridge CatCart ${ }^{\circledR}, h=70 \mathrm{~mm}$, 100 i.d. $=3 \mathrm{~mm}$ ) heated by an isothermal heating jacket controlled by a Peltier unit (Scheme 2). The catalyst $(269 \mathrm{mg}$, sieve fraction = $0.2-0.4 \mathrm{~mm}$ ) was loaded into the reactor and heated at $363 \mathrm{~K}$. Thereafter, the reaction was started by admitting a 2 wt.\% GLY aqueous solution to the catalytic bed by means of an on-line 105 HPLC pump at a flow rate of $0.3 \mathrm{~cm}^{3} \mathrm{~min}^{-1}$.

GLY and glycolic acid (GA) were isolated by highperformance liquid chromatography (HPLC) in a Merck LaChrom system equipped with a Biorad Aminex HPX-87H column heated at $308 \mathrm{~K}$ and a refractive index detector (Hitachi ${ }_{110}$ Chromaster model 5450) set at $303 \mathrm{~K}$, using an aqueous eluent of $0.005 \mathrm{M} \quad \mathrm{H}_{2} \mathrm{SO}_{4} \quad(\mathrm{pH} 2.30)$ flowing at $0.6 \mathrm{~cm}^{3} \mathrm{~min}^{-1}$. Quantification was obtained by integration of their respective peaks using butan-2-one (Merck, 99.5\%) as an internal standard. 


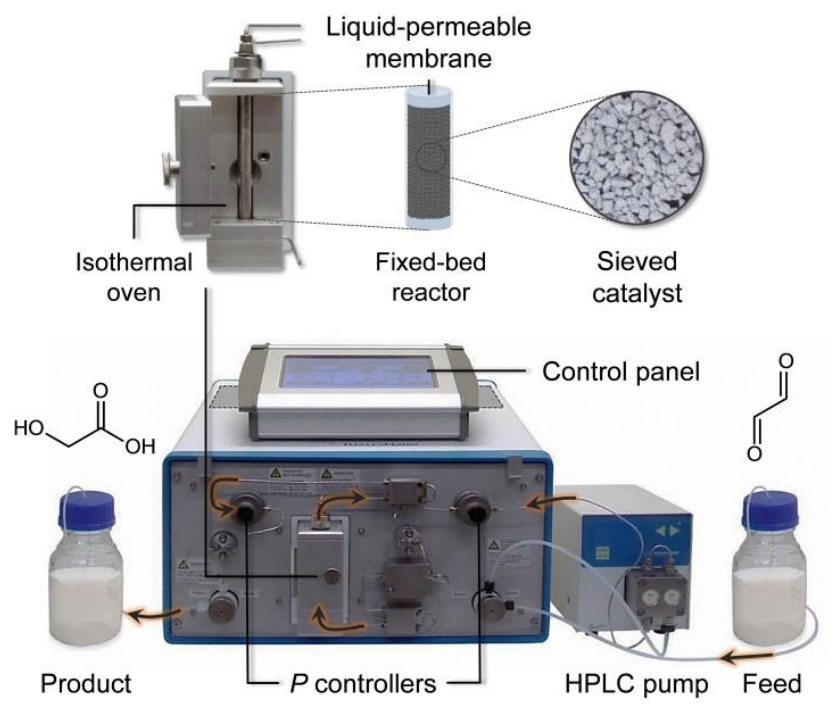

Scheme 2 Illustration of the catalytic set-up used for the continuous-flow isomerisation of glyoxal.

Alkyl glycolates were analysed using a gas chromatograph (GC, HP 6890) equipped with an HP-5 capillary column and a flame 5 ionisation detector. Methyl glycolate (Sigma-Aldrich, 98\%), ethyl glycolate (Sigma-Aldrich, 98\%), and $n$-butyl glycolate (SigmaAldrich, 90\%) were employed as references. He was used as the carrier gas (flow rate $=4.3 \mathrm{~cm}^{3} \mathrm{~min}^{-1}$, pressure $=1.4$ bar) and an injection volume of $0.3 \mu \mathrm{L}$ was applied. The initial temperature 10 of $328 \mathrm{~K}$ was held for $2 \mathrm{~min}$ before heating to $473 \mathrm{~K}$ $\left(30 \mathrm{~K} \mathrm{~min}^{-1}\right)$. The alkyl glycolate yields were determined using diethyl malonate (Sigma-Aldrich, 99\%) as an internal standard. The GLY conversion was calculated as the mole of GLY reacted divided by the mole of GLY fed, whereas the GA/alkyl glycolate 15 yields as the mole of GA/alkyl glycolate formed divided by the initial moles of GLY. Both conversion and yields are expressed as a percentage.

\section{Results and discussion}

\subsection{Commercial USY zeolites and galliated samples}

20 Based on the mechanistic information mentioned in the introduction and in order to evaluate the role of the type of acidity in the GLY isomerisation, the reaction was initially investigated over commercially available USY zeolites. These aluminosilicates possess Brønsted as well as Lewis acidity, the 25 latter originating from the extraframework aluminium (EFAl) species generated by steaming and acid leaching of the pristine $\mathrm{Y}$. Various USY zeolite catalysts were selected in order to span through a wide range of EFAl amounts. The evolution of the concentration of the components of the reaction mixture was first 30 studied over CBV712 (H-USY, $\mathrm{Si} / \mathrm{Al}=6$ ), a material characterised by substantial Lewis acidity. The conversion of GLY progressively increased with time attaining a value of $66 \%$ after $48 \mathrm{~h}$ (Fig. 1). Simultaneously, the GA yield rose up to $60 \%$. Remarkably, no by-products were detected, in contrast to the case 35 of the aqueous-phase isomerisation of dihydroxyacetone in which the pyruvaldehyde intermediate, i.e. the GLY analogue, was prone to degradation into acetol, pyruvic acid, and formaldehyde

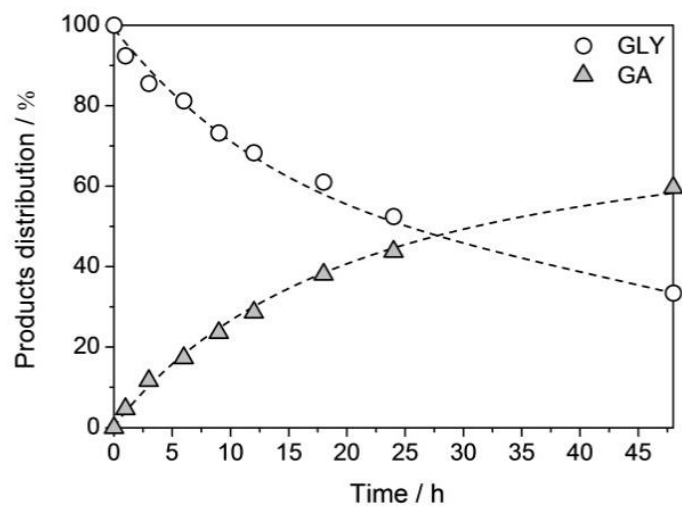

Fig. 1 Concentration profiles of the reactant and product during the 40 conversion of GLY in water over CBV712 at $363 \mathrm{~K}$.

over Brønsted-acid sites.[25] Impurities present in the substrate, including formic acid, acetic acid, and ethylene glycol, account for the remaining fraction to close the carbon balance. Since separation of bio-based molecules is often regarded as a strong 45 contributor to the process cost, the absence of by-products is extremely advantageous in the perspective of a large-scale implementation. In order to compare the performance of the zeolites, $18 \mathrm{~h}$ was selected as the reaction time as it appeared as a good compromise between the yield of GA and the time needed ${ }_{50}$ for its production. Before $18 \mathrm{~h}$, the gain in the glycolic acid yield per unit time is considerable while it becomes progressively less and less pronounced thereafter (Fig. 1). While the GA selectivity remained unaffected (above 90\%), the GLY conversion and the GA yield increased with the EFAl content (Fig. 2), reaching a 55 maximum value of 62 and $57 \%$, respectively, over the severelysteamed and most EFAl-rich CBV600 (Table 1, entry 3). Notably, the EFAl-free CBV760, CBV780, and HSZ390 converted GLY to GA to the level of only $2-6 \%$. These results clearly demonstrate Lewis-acid sites as the catalytic centres for ${ }_{60}$ the reaction, while the cooperative effect of Brønsted acid sites seems negligible. This is further supported by $(i)$ the comparable trend observed for the Lewis-acid catalysed production of ethyl lactate over a similar set of USY zeolites,[19b] and (ii) the inactivity of a purely Brønsted-acidic material, Amberlyst-15 65 (Table 1, entry 2). This conclusion corroborates the DFT

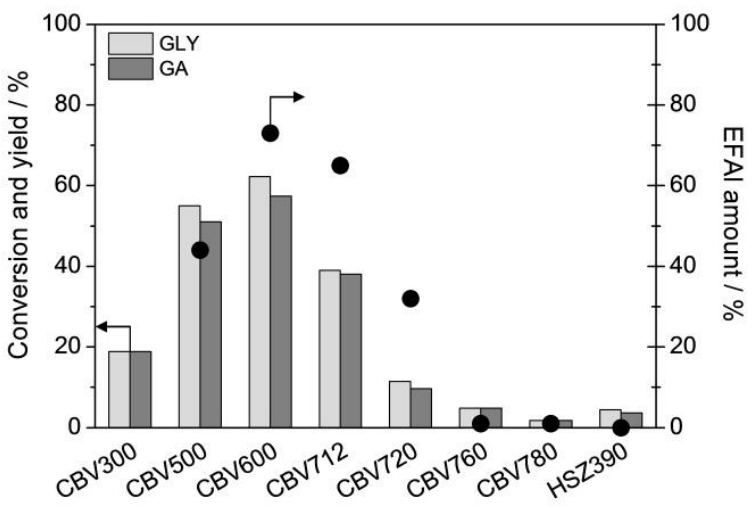

Fig. 2 GLY conversion and GA yield over various commercially available USY zeolites at $363 \mathrm{~K}$ and $18 \mathrm{~h}$. The EFAl amount in the samples was derived from ref. 26. 
Table 1 Composition, textural properties, and main catalytic data obtained after $18 \mathrm{~h}$ at $363 \mathrm{~K}^{a}$

\begin{tabular}{|c|c|c|c|c|c|c|c|c|}
\hline Entry & Catalyst $^{b}$ & $\begin{array}{c}\mathrm{Si} / \mathrm{Al}^{c} \\
\left(\mathrm{~mol} \mathrm{~mol}^{-1}\right)\end{array}$ & $\begin{array}{l}\text { Metal content }{ }^{c} \\
\quad(\text { wt. } \%)\end{array}$ & $\begin{array}{c}S_{\text {meso }}{ }^{d} \\
\left(\mathrm{~m}^{2} \mathrm{~g}^{-1}\right)\end{array}$ & $\begin{array}{c}V_{\text {micro }}{ }^{d} \\
\left(\mathrm{~cm}^{3} \mathrm{~g}^{-1}\right)\end{array}$ & $\begin{array}{c}\text { GLY conversion } \\
(\%)\end{array}$ & $\begin{array}{c}\text { GA yield } \\
(\%)\end{array}$ & $\begin{array}{c}\text { GA selectivity } \\
(\%)\end{array}$ \\
\hline 1 & Blank & - & - & - & - & 1 & 1 & 100 \\
\hline 2 & Amberlyst-15 & - & - & 41 & 0 & 1 & 1 & 100 \\
\hline 4 & CBV720-P & 17 & - & 128 & 0.29 & 11 & 10 & 91 \\
\hline 5 & CBV720-ATGa & 10 & 7.5 & 186 & 0.20 & 80 & 77 & 96 \\
\hline 6 & CBV720-ATGa [373K] & 10 & 7.5 & 186 & 0.20 & 97 & 91 & 94 \\
\hline 7 & Ti-MFI & - & n.d. & 40 & 0.14 & 65 & 59 & 91 \\
\hline 10 & MFI-P & 961 & - & 53 & 0.17 & 4 & 3 & 100 \\
\hline 11 & MFI-ATSn & - & 2.55 & 118 & 0.10 & 96 & 90 & 94 \\
\hline 12 & MFI-ATSn [6h] & - & 2.55 & 118 & 0.10 & 73 & 69 & 95 \\
\hline
\end{tabular}

${ }^{a}$ Reaction conditions: $W_{\text {cat }}=80 \mathrm{mg}, W_{\mathrm{H} 2 \mathrm{O}}=3.80 \mathrm{~g}, W_{\mathrm{GLY}}=0.20 \mathrm{~g} .{ }^{b} \mathrm{P}=$ parent, $\mathrm{AT}=$ alkaline treatment with $0.3 \mathrm{M}$ NaOH in the presence of a gallium 5 (Ga) or tin (Sn) source. ${ }^{c}$ Determined by ICP-OES. Metals comprise $\mathrm{Sn}$ or Ga. ${ }^{d}$ Determined by the $t$-plot method.

prediction of a more favourable isomerisation mechanism based on a 1,2-hydride shift rather than dehydrationtautomerisation.[16] The reaction pathway is demonstrated by NMR spectroscopic studies in the following.

10 Based on these findings, with the aim of attaining an optimised catalytic system for the production of GA, Lewis-acidic zeolites already reported in the literature as well as a novel material were investigated. First, post-synthetic modification of the USY framework by alkaline-assisted galliation was considered, which 15 already proved to render highly selective catalysts for the isomerisation of dihydroxyacetone in alcoholic media.[19b,c] The best performing galliated zeolite reported in that work, obtained by treatment of CBV720-P (Table 1, entry 4) with $0.2 \mathrm{M} \mathrm{NaOH}$ and $0.04 \mathrm{M} \mathrm{Ga}\left(\mathrm{NO}_{3}\right)_{3}$, displayed improved results: $74 \%$ GLY 20 conversion and $71 \%$ GA yield. Further tuning of the concentration of both the base $(0.3 \mathrm{M})$ and the gallium salt $(0.05 \mathrm{M})$ in the zeolite treatment enabled to increase the amount of gallium incorporated in the USY framework from $c a$. 6 to $7.5 \mathrm{wt} . \%$, still largely preserving the original crystallinity of the 25 sample (Fig. SI1). This optimised CBV720-ATGa catalyst (Table 1, entry 5) exhibited enhanced GLY conversion and GA yield, i.e. 80 and $77 \%$, respectively, which thus surpassed by $c a$. $20 \%$ those obtained over the best commercial aluminosilicate identified (CBV600). Remarkably, raising the temperature by 30 only $10 \mathrm{~K}$ enabled to reach almost full GLY conversion and a GA yield as high as $91 \%$ (Table 1, entry 6).

\subsection{Ti- and Sn-based MFI zeolites}

As the next step, tin- and titanium-based zeolites were 35 considered. Isomorphously-substituted zeolites prepared hydrothermally and containing these metals are expected to be superior catalytic materials owing to the intrinsically stronger Lewis acidity and structural uniformity of the tin and titanium species, which have already enabled very fast conversion rates of 40 trioses, pentoses, and hexoses.[17] With regards to tin-containing zeolites, Sn-BEA synthesised in fluorine media exhibited unrivalled performances in these Lewis-acid catalysed isomerisations.[17] Nevertheless, since its complex synthesis demonstrated irreproducible even at the lab-scale level, materials

45 based on the MFI framework, which can be obtained in much more straightforward manner, were preferred for evaluation in the transformation of GLY. Furthermore, even if a similar or higher activity of Sn-BEA compared to Sn-MFI for the isomerisation of $\mathrm{C}_{2}$ substrates cannot be excluded, there is 50 indication in the literature that the latter zeolite has a better isomerisation ability than the former for smaller bio-derived substrates ( $\mathrm{C}_{3}$ molecules).[27] The textural properties, chemical composition, and catalytic performance of the prepared Ti-MFI and Sn-MFI are reported in Table 1 (entries 7 and 8). ${ }_{55}$ Surprisingly, the GLY conversion and GA yield over the former solid only reached $65 \%$ and $59 \%$ respectively, thus being similar to the performance of CBV600. The limited activity of this catalyst might relate to the additional presence of titanium in form of extraframework $\mathrm{TiO}_{x}$ species, which are unavoidably 60 generated to some extent during synthesis and calcination, as confirmed by UV-Vis spectroscopy of our sample (Fig. SI2). In contrast, Sn-MFI yielded $90 \%$ of GA at full GLY conversion after only $6 \mathrm{~h}$ (Table 1 , entry 9), therefore confirming the exceptional characteristics of tin-based materials.

65 Due to the typically long synthesis time and the need for HF, no industrially applicable protocol has been developed so far for the preparation of tin-containing zeolites (e.g. Sn-BEA and SnMFI).[17d, 28] Therefore, the availability of an analogous material obtained via an alternative and more scalable route 70 would be highly relevant. We first wondered whether the extrapolation of recently reported protocols for the preparation of a highly active Sn-beta, could be instrumental for the purpose. Such methods comprise dealumination of an aluminium-rich zeolite beta followed by tin incorporation by either solid-state ion 75 exchange or wet impregnation with a $\mathrm{SnCl}_{4}$ solution in isopropanol.[29] As the demetallation step, common to the two routes, requires the use of concentrated and, thus, highly corrosive nitric acid and the solid-state reaction may not be applicable at a large scale, we rather conceived to attempt the 80 preparation of a Sn-MFI by alkaline-assisted metallation. This approach involves the creation of defects in the zeolite structure by the action of hydroxide anions and their healing with tin cations in one pot under mild conditions and holds brighter premises for industrial viability, as post-synthetic alkaline-based 85 treatments of commercial zeolites have already been successfully demonstrated at the kilogram scale.[30] Nevertheless, it should be underlined that the chemical challenge associated with this strategy is considerable as only the incorporation of trivalent 

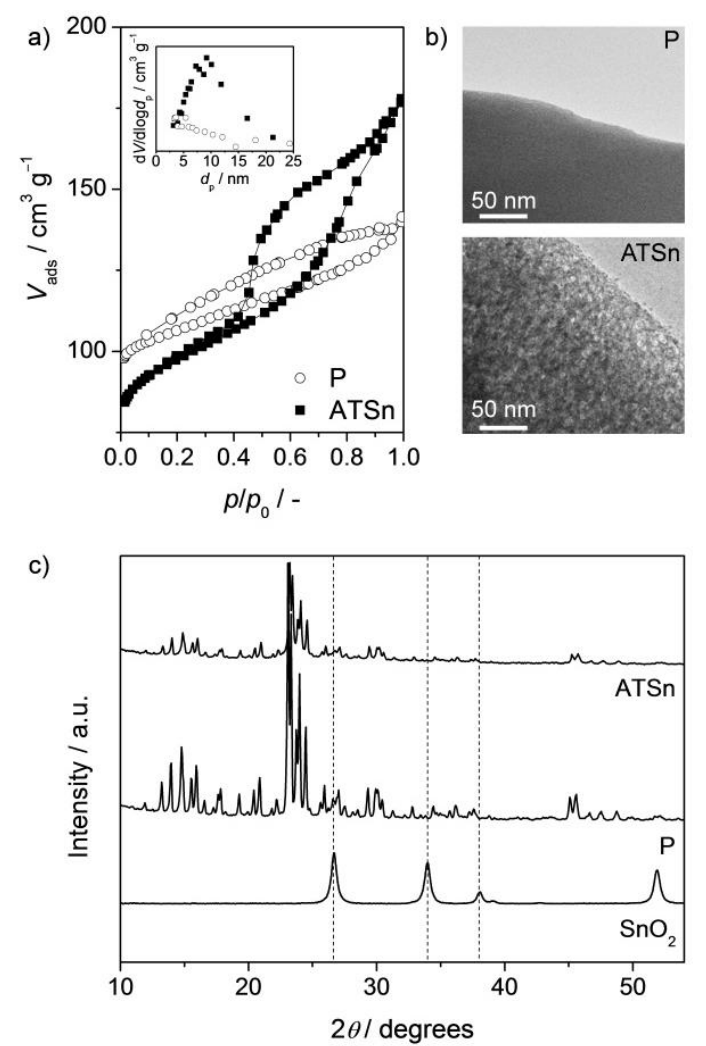

Fig. 3 (a) $\mathrm{N}_{2}$ sorption isotherms, (b) transmission electron micrographs and, (c) X-ray diffraction patterns of MFI in parent form and after alkaline treatment in the presence of $\mathrm{Sn}\left(\mathrm{SO}_{4}\right)_{2}$. The inset in ' $\mathrm{a}$ ' depicts the pore size distribution of the two samples.

5 metal ions $\left(\mathrm{M}=\mathrm{Al}^{3+}\right.$ and $\left.\mathrm{Ga}^{3+}\right)$, existing as negatively charged hydroxide species $\mathrm{M}(\mathrm{OH}) 4^{-}$in strong alkaline media, has been demonstrated so far.[19] Not only tin cations would possess a different charge (either 2 or 4), but also the hydroxides correspondingly formed in the basic solution are very poorly 10 soluble and instantaneously from a precipitate. Still, taking into account the relatively small quantity of tin required to generate a highly active catalyst (1.7 wt.\% Sn in Sn-MFI), it was thought that even a tiny amount of $\mathrm{Sn}(\mathrm{OH})_{n} q^{-}$in solution could be a sufficient source of the metal. In order to enable a direct 15 comparison with Sn-MFI, the MFI-type silicalite-1 zeolite (denoted hereafter as MFI-P) was selected as the parent material. Modification was performed in the presence of a $0.3 \mathrm{M} \mathrm{NaOH}$ solution saturated with $\mathrm{Sn}(\mathrm{OH})_{n}{ }^{q-}$, prepared using $\mathrm{Sn}\left(\mathrm{SO}_{4}\right)_{2}$ as the precursor. The composition and textural properties of the 20 resulting sample, MFI-ATSn, are summarised in Table 1 (entry 11). Elemental analysis of the solid and the filtrate after synthesis indicated the incorporation of $2.55 \mathrm{wt} . \%$ tin in the zeolite and a tin insertion efficiency upon the alkaline-assisted process equal to 13\%. The uptake at mid-high relative pressures in the $\mathrm{N}_{2}$ 25 isotherms indicated the development of intra-particle mesoporosity $\left(118 \mathrm{~m}^{2} \mathrm{~g}^{-1}\right.$, pore size distribution centred at $\left.8 \mathrm{~nm}\right)$, which was further evidenced by transmission electron microscopy (Figs. 3a, b). No additional phase to the zeolite could be visualised by the latter technique, which would suggest the ${ }_{30}$ presence of tin as $\mathrm{SnO}_{2}$. High-angle $\mathrm{X}$-ray diffraction analysis evidenced the retention of the MFI structure upon metallation and the lack of diffraction peaks specific to tin oxide, supporting the

absence of this phase, at least in form of large particles (Fig 3c). Remarkably, MFI-ATSn exhibited a nearly identical performance 35 with respect to Sn-MFI, i.e. $96 \%$ GLY conversion and 90\% GA.

Since this result was attained after a longer reaction time (18 versus $6 \mathrm{~h}$ ), the catalytic properties of the tin sites introduced by post-synthetic metallation appeared slightly inferior to those of the hydrothermally-synthesised catalyst. This difference was 40 more adequately quantified based on the turnover frequency (TOF), calculated as the mole of product formed per hour and mole of Sn after $30 \mathrm{~min}$ of reaction, and the catalyst productivity, determined as the $\mathrm{g}$ of product formed per hour and per $\mathrm{kg}$ of catalyst at a conversion level of $20 \%$. Lower values of TOF and 45 productivity were indeed obtained for MFI-ATSn $\left(\mathrm{ca} .65 \mathrm{~h}^{-1}\right.$ and $\left.1060 \mathrm{~g} \mathrm{~kg}^{-1} \mathrm{~h}^{-1}\right)$ than for Sn-MFI (ca. $191 \mathrm{~h}^{-1}$ and $2842 \mathrm{~g} \mathrm{~kg}^{-1} \mathrm{~h}^{-1}$ ). An in-depth investigation of the main features of the novel catalyst was performed aiming at deriving fundamental understanding of differences and similarities between the 50 incorporation of tin by bottom-up and top-down strategies.

FTIR spectroscopy of adsorbed pyridine was applied to provide insights into the amount and the strength of the Lewisacid sites introduced. Fig. 4a depicts the infrared spectra of MFIATSn and Sn-MFI after pyridine adsorption at room temperature 55 and subsequent desorption at 473, 523, 573, and $623 \mathrm{~K}$. The amount of Lewis-acid sites in the samples was calculated by integration of the signal at $1454 \mathrm{~cm}^{-1}$ in the spectrum collected after degassing at $473 \mathrm{~K}$, as this temperature is sufficient to remove the physisorbed pyridine and the pyridine bonded to the 60 silanols while preserving the pyridine chemisorbed on tin centres. A 1.8 times higher concentration of acid sites was found for MFI-
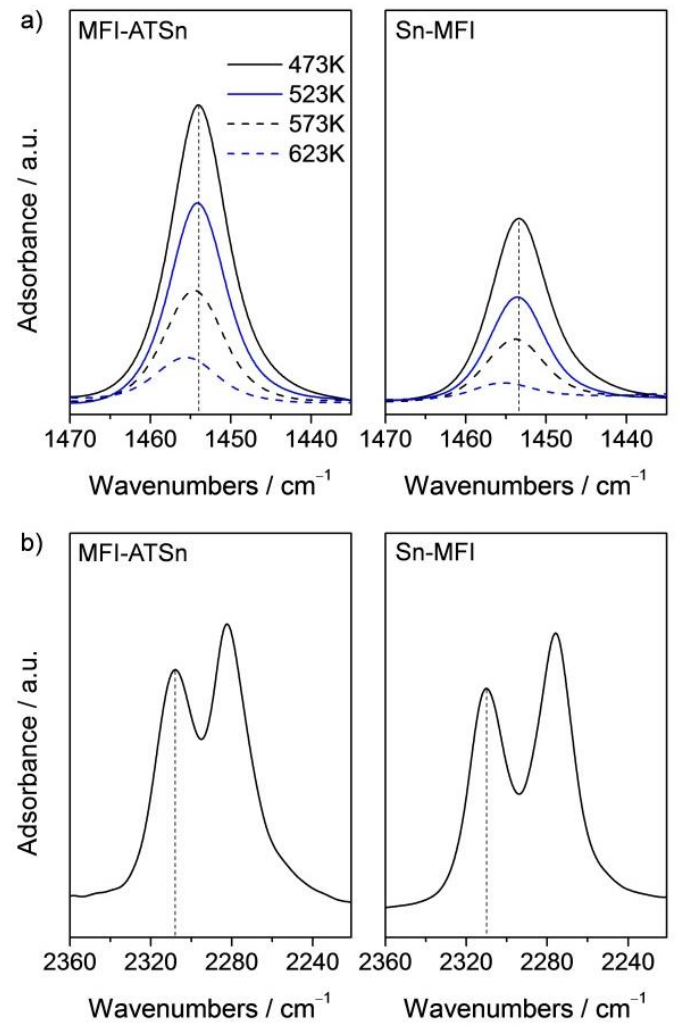

Fig. 4 FTIR spectra of MFI-ATSn and Sn-MFI after (a) pyridine adsorption followed by evacuation at different temperatures and (b) d3acetonitrile adsorption followed by evacuation at room temperature. 

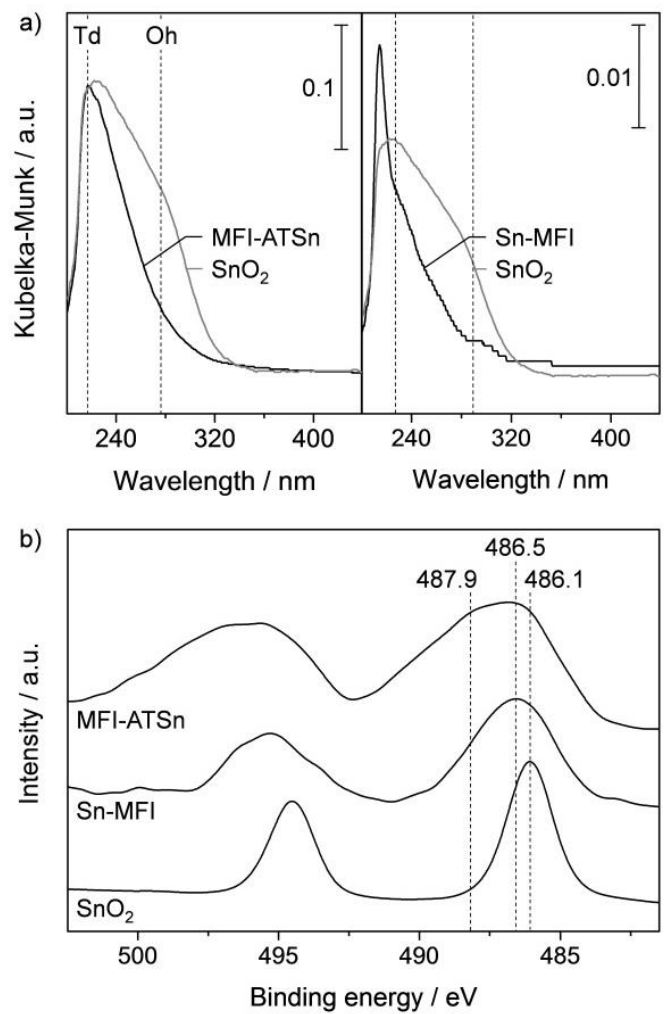

Fig. 5 (a) Diffuse reflectance UV-Vis and (b) Sn $3 d$ core-level XPS spectra of $\mathrm{SnO}_{2}$, Sn-MFI, and MFI-ATSn.

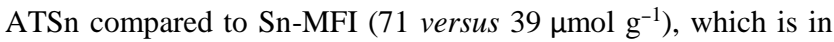
good agreement with the 1.5 times higher tin content in the 5 former catalyst, considering the experimental errors associated with the two characterisation techniques. This finding implies that all of the Sn centres are accessible to pyridine and, thus, that each metal atom can potentially act as a catalytic site. The strength of the Sn sites was determined by evaluating the ratio of

10 the integrals of the same absorption band in the spectra collected after pyridine desorption at 473 and $573 \mathrm{~K}$, in line with previous reports.[29b] Values of 0.36 and 0.31 were obtained for MFIATSn and Sn-MFI, respectively, thus pointing to a relatively similar Lewis-acid strength of the Sn centres derived from the 15 two synthetic methods. In order to corroborate this result, the samples were probed with a molecule with weaker basicity, d3acetonitrile (Fig. 4b). Upon chemisorption on a Lewis-acid site, the band associated with the stretching of the nitrile bond undergoes a shift in position which has been reported to strongly 20 depend on the strength of the site $[28,31,32]$. In agreement with previous evidence, the signal for $\mathrm{d} 3$-acetonitrile chemisorbed on Sn-MFI appeared at $2310 \mathrm{~cm}^{-1}$, indicating the presence of framework Sn atoms [28]. The band obtained for MFI-ATSn was very similar and positioned at only $2 \mathrm{~cm}^{-1}$ lower frequency. These 25 evidences confirm the equality of the acid strength of the tin sites in the two catalysts.

As the difference in catalytic activity of the two materials cannot be rationalised based on their acid properties, diffuse reflectance UV-Vis spectroscopy and XPS were employed to 30 shed light onto the structure of the tin species located in these zeolites (Fig. 5). The UV-Vis spectrum of MFI-ATSn displays a peak at $217 \mathrm{~nm}$, generally assigned to an electronic transition of isolated $\mathrm{Sn}(\mathrm{IV})$ species coordinated to four oxygen atoms in the zeolite framework.[33,34] No evidence for hexa-coordinated ${ }_{35}$ polymeric Sn species was found, which would typically give rise to a band at $280 \mathrm{~nm}$, as observed for the reference material $\mathrm{SnO}_{2}$.[33,34] The peak related to tetra-coordinated species appeared sharper in the case of Sn-MFI,[35] highlighting the greater uniformity of the Lewis-acid sites incorporated through 40 hydrothermal synthesis and the larger distribution in the environments of the Lewis-acid centres introduced via postsynthetic metallation. When considering the $\mathrm{Sn} 3 d$ core-level XPS spectra, hydrothermally-synthesised Sn-MFI produced a signal at $c a$. $486.5 \mathrm{eV}$, which matches that obtained from 45 hydrothermally-synthesised Sn-beta.[36]. The sample prepared by alkaline-assisted metallation displayed a broader spectral feature comprising the same contribution as found for Sn-MFI as well as at least another component at higher binding energy ( $c a$. $487.9 \mathrm{eV})$, suggesting that $\mathrm{Sn}(\mathrm{IV})$ atoms in the material have non${ }_{50}$ equivalent environments. It can be speculated that a fraction of tin centres is totally integrated in the framework (alike in $\mathrm{Sn}$ MFI), while a fraction might be only partially incorporated. The presence of extra-framework tin species in an amount below the detection limit of the techniques used cannot be discarded.

55 Besides for differences arising from the distinct preparation routes, it might be possible that silicalite-1 could not fully accommodate the tin atoms in framework positions due to the higher metal loading in the final catalyst compared to Sn-MFI. The heterogeneity of the Sn sites in MFI-ATSn is expected to 60 determine the slight inferiority of MFI-ATSn in GLY isomerisation. Nonetheless, the major similarity of the tin sites obtained by the two methods remains quite striking.

\subsection{Elucidation of the reaction mechanism by NMR 65 Spectroscopy}

In order to unravel the reaction mechanism, the conversion of GLY was performed over MFI-ATSn in deuterated water and the mixture analysed by ${ }^{13} \mathrm{C}$ NMR spectroscopy. As stated above, the isomerisation of GLY to GA can theoretically follow two main 70 routes, being a Brønsted-acid catalysed dehydration or a Lewisacid catalysed 1,2-hydride shift (Scheme 3). Even though the nature of the centres in MFI-ATSn is substantially Lewis-acidic, and thus the latter mechanism should obviously be favoured, the presence of a minor component of Brønsted acidity, and therefore 75 the occurrence of the former mechanism, cannot be fully excluded. In fact, it was demonstrated that Lewis-acid tin sites in $\mathrm{Sn}$-BEA are partially hydrolysed, i.e. $\mathrm{Sn}-\mathrm{OH}$, in the presence of water.[37] While the dehydration route involves the reaction of the dihydrate form of GLY, the 1,2-hydride shift occurs on the 80 monohydrate form. A recent study indicated that both hydrate

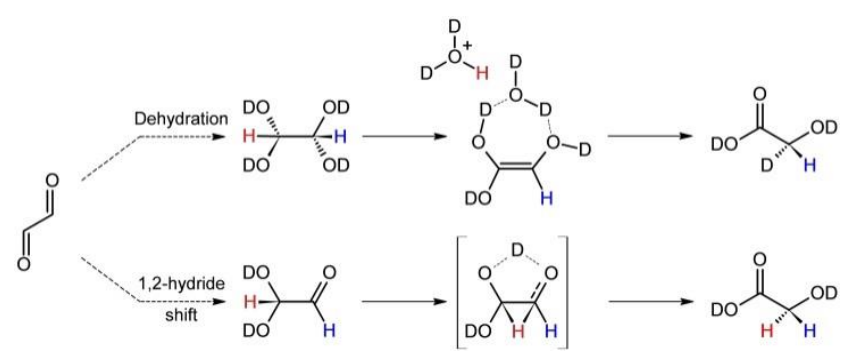

Scheme 3 Possible reaction pathways for the catalytic isomerisation of GLY to GA. 


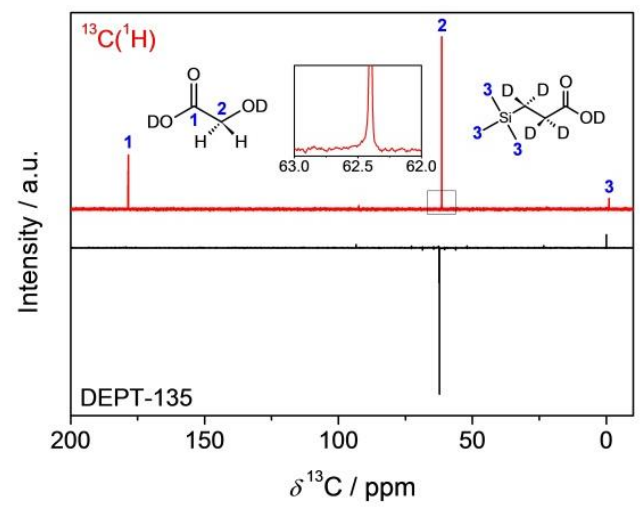

Fig. $6{ }^{13} \mathrm{C}$ NMR spectra of the reaction mixture obtained at $363 \mathrm{~K}$ and $18 \mathrm{~h}$ over MFI-ATSn.

forms are present in relatively similar quantities in water,[38] although ${ }^{1} \mathrm{H}$ NMR spectroscopy experiments evidenced the 5 presence of only one peak corresponding to the monohydrate compound.[16] The dehydration mechanism comprises the formation of a double bond by syn-elimination of water and subsequent tautomerisation of the resulting enol intermediate into its keto-isomer, that is GA. The 1,2-hydride shift mechanism 10 relies on the concomitant oxidation of the $\alpha$-hydroxyl and reduction of the carbonyl group, that is referred to as an intramolecular Meerwein-Ponndord-Verley reduction Oppenauer oxidation (MPVO redox). Consequently, while the dehydration mechanism is expected to introduce deuterium in the carbon 15 backbone, no deuterium incorporation should occur via the 1,2hydride shift. Fig. 6 represents the $1 \mathrm{D}{ }^{13} \mathrm{C}$ and DEPT-135 NMR spectra of the mixture obtained upon conversion of GLY over MFI-ATSn after $18 \mathrm{~h}$. The ${ }^{13} \mathrm{C}$ NMR spectrum displays two main peaks centred at 62.5 and $178.5 \mathrm{ppm}$, respectively associated with 20 the secondary and quaternary carbon of GA. A small peak at 93.4 ppm, due to the dihydrate form of GLY, is also visible. Interestingly, the peak at $62.5 \mathrm{ppm}$ does not show any triplet structure caused by a ${ }^{1} \mathrm{~J}_{\mathrm{DH}}$-coupling to a directly attached deuterium nucleus (see inset in Fig. 6, top). The absence of ${ }_{25}$ deuterium in the backbone was further evidenced by the $1 \mathrm{D}{ }^{13} \mathrm{C}$ DEPT-135 spectrum, in which the methyl groups of sodium 3(trimethylsilyl)propionoate-2,2,3,3-d4 produce a reference peak to correctly assign the polarity of the signals. The fact that the peak centred at $62.5 \mathrm{ppm}$ is negative undoubtedly confirms the 30 sole presence of $\mathrm{CH}_{2}$ species, and thus the exclusivity of the 1,2hydride shift mechanism.

\subsection{Assessment of industrially relevant aspects of Sn-based zeolites}

35 In order to assess the potential of MFI-ATSn for a practical application, its stability was evaluated by means of recyclability and hot-filtration studies. In the former case, the GLY conversion and GA yield were monitored upon direct reuse of the catalyst, i.e. without any intermediate regeneration, in five subsequent 40 catalytic runs (Fig 7a). Remarkably, no loss of activity was observed and the GA yield decreased by only $5 \%$ by the fifth run. The original performance could be fully recovered by calcination $\left(823 \mathrm{~K}, 5 \mathrm{~K} \mathrm{~min}^{-1}, 5 \mathrm{~h}\right.$, static air) of the sample at this stage, as the outcome of the sixth experiment indicates. To exclude that tin 45 leaching took place, the catalyst was filtered off at the reaction a)

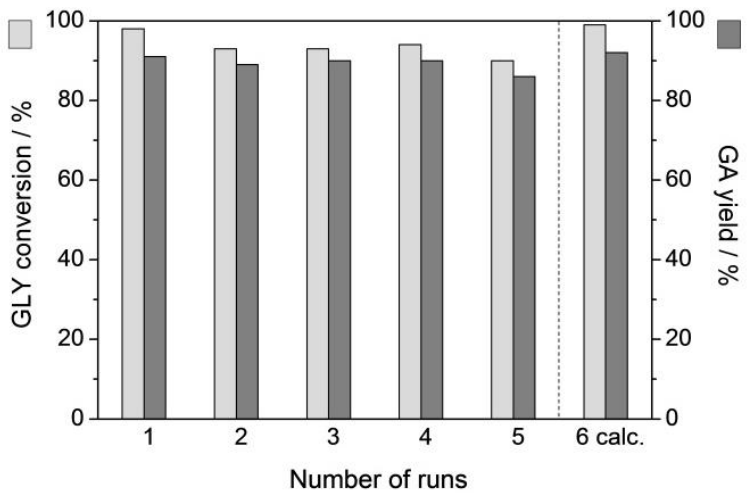

b)

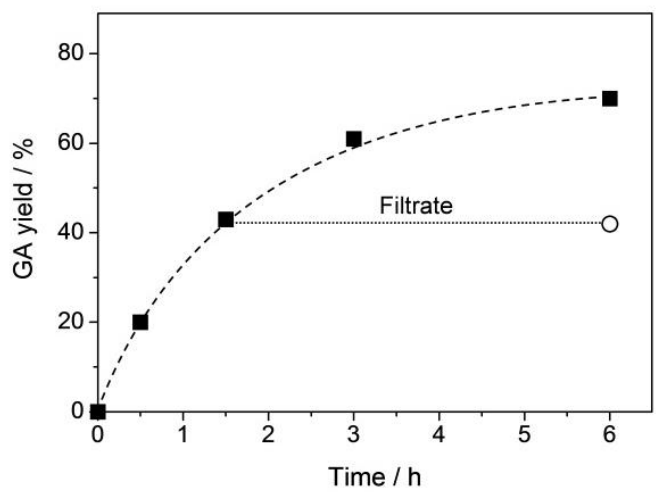

Fig. 7 (a) GLY conversion and GA yield at $363 \mathrm{~K}$ and $18 \mathrm{~h}$ upon reuse in consecutive catalytic runs and (b) hot-filtration test over MFI-ATSn.

temperature from the mixture obtained after $1.5 \mathrm{~h}$ and the filtrate was allowed to react for $4.5 \mathrm{~h}$. As illustrated in Fig. 7b, no further 50 production of GA was detected after removal of the catalyst, thus confirming the truly heterogeneous nature of the catalyst.

Based on these results, the extrapolation from a batch to continuous-flow mode was investigated, since the latter is widely preferred for the production of bulk chemicals, such as GA. A 552 wt.\% GLY aqueous solution was allowed to flow at $0.3 \mathrm{~cm}^{3} \mathrm{~min}^{-1}$ during $8 \mathrm{~h}$ through a catalytic bed $\left(0.5 \mathrm{~cm}^{3}\right)$ of MFI-ATSn heated at $363 \mathrm{~K}$. Under these conditions, both the GLY conversion and GA selectivity remained stable at the remarkable levels of 40 and $96 \%$, respectively (Fig. 8). We would ${ }_{60}$ like to stress the relevance of this result, as the wide majority of chemocatalytic systems for the production of high-volume chemicals in the field of biomass conversion have been so far

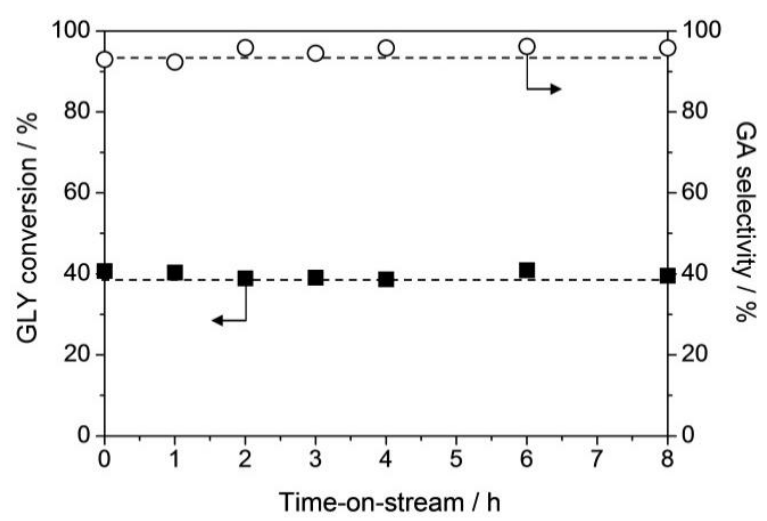

Fig. 8 GLY conversion and GA selectivity in a continuous-flow experiment over MFI-ATSn at $363 \mathrm{~K}$. 


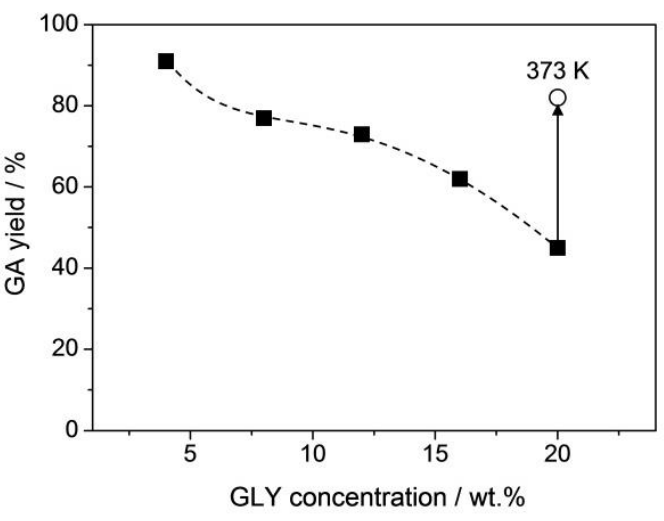

Fig. 9 GLY feed concentration versus GA yield over MFI-ATSn.

demonstrated limitedly to the batch operation.

Finally, the possibility to attain a more industrially significant process productivity was explored by evaluating the catalyst 5 response to the application of a higher feed concentration. Tests were conducted batch-wise in which the catalyst amount was kept constant (ca. $80 \mathrm{mg}$ ) each time, while the GLY concentration was raised up to five-fold, i.e. $20 \mathrm{wt}$ \%. As shown in Fig. 9, the GA yield progressively decreased to $45 \%$ upon utilisation of less and 10 less diluted solutions. Nevertheless, raising the reaction temperature by only $10 \mathrm{~K}$ enabled to substantially restore the GA yield $(81 \%)$ upon the use of the most concentrated feed.

\subsection{Production of alkyl glycolates over Sn-based zeolites}

15 As the substitution of the water medium by an alcohol enabled the direct conversion of dihydroxyacetone into the corresponding ester derivatives of lactic acid,[19b,c] we approached the preparation of methyl, ethyl, and butyl glycolates following the same strategy (Scheme 1). While the latter two compounds find 20 widespread application as plasticisers, methyl glycolate represents an intermediate in the industrial manufacture of ethylene glycol. [20] Table 2 reports the performances of MFIATSn and Sn-MFI in the three alcoholic solvents after a reaction time of 18 and $6 \mathrm{~h}$, respectively. The yield of the alkyl glycolates 25 reached a maximum of $83 \%$ for the methyl ester, thus being overall lower compared to the case of GA (91\%). Specifically, the higher the length of the alcohol; the lower was the yield of the corresponding alkyl glycolate. Sn-MFI was slightly superior to MFI-ATSn in methanol, whereas an inverse trend was observed 30 in ethanol and $n$-butanol. The latter is a likely consequence of the more pronounced benefit of the presence of a mesoporous network in MFI-ATSn when dealing with larger molecules. In

Table 2 GLY conversion over MFI-ATSn and Sn-MFI in various alcohols $^{a}$

\begin{tabular}{cccc}
\hline Solvent & Catalyst & $\begin{array}{c}\text { GLY conversion } \\
(\%)\end{array}$ & $\begin{array}{c}\text { Glycolate yield } \\
(\%)\end{array}$ \\
\hline \multirow{2}{*}{ methanol } & MFI-ATSn & 85 & 82 \\
& Sn-MFI & 85 & 83 \\
ethanol & MFI-ATSn & 73 & 69 \\
& Sn-MFI & 70 & 68 \\
$n$-butanol & MFI-ATSn & 53 & 53 \\
& Sn-MFI & 49 & 45
\end{tabular}

${ }_{35}{ }^{a}$ Reaction conditions: $W_{\text {cat }}=80 \mathrm{mg}, W_{\text {alcohol }}=3.80 \mathrm{~g}, W_{\mathrm{GLY}}=0.20 \mathrm{~g}, t=$ 6 or $18 \mathrm{~h}$ for Sn-MFI or MFI-ATSn, respectively. line with the essentially pure Lewis-acid character of the catalysts, no evidence for the Brønsted-acid catalysed alkylation of glyoxal was found. The competitive formation of pyruvic 40 aldehyde dialkyl acetals was also suppressed in the case of dihydroxyacetone isomerisation over tin-based zeolites.

\section{Conclusions}

A chemocatalytic process for the valorisation of glyoxal, an undesired oxygenated component of pyrolysis oil, was 45 investigated in this study. Its aqueous-phase isomerisation over Lewis-acid zeolites was put forward as an efficient way to renewably produce glycolic acid, a relevant building block for polymers manufacture. Spectroscopic investigations indicated that the reaction mechanism relies on a 1,2-hydride shift. Tin50 containing materials demonstrated the best catalysts for this reaction, yielding more than $90 \%$ of the target product in short times at only $363 \mathrm{~K}$ without forming any by-products. Besides confirming the extraordinary Lewis-acid properties of hydrothermally-synthesised Sn-MFI, our work uncovered a novel 55 zeolite displaying similar characteristics, which was generated by simple alkaline treatment of silicalite- 1 in the presence of a tin salt. The catalyst was shown to be recyclable and to act in truly heterogeneous manner. Furthermore, remarkable results were obtained in its application for the continuous-flow production of 60 glycolic acid. The generation of a set of industrially relevant alkyl glycolates by isomerisation of glyoxal in different alcoholic solvents was also successfully achieved on the tin-based zeolites. Overall, the proposed process combines: $(i)$ the use of a renewable and waste-type feedstock, (ii) the application of mild ${ }_{65}$ conditions, thus limiting the energy requirements, (iii), the use of green solvents, (iv) the absence of by-products, and $(v)$ the intrinsic advantages of a heterogeneous catalyst, which is prepared according to an industrially scalable protocol, and thus appears to hold a great potential to replace or substantiate the 70 current environmentally-unfriendly process for the production of glycolic acid and to establish a one-pot methodology for the generation of its alkyl esters derivatives.

\section{Acknowledgments}

This work was supported by the Swiss National Science 75 Foundation (Project Number 200021-140496). The Electron Microscopy Centre of ETH Zurich is acknowledged for the use of their facility. Dr. S. Mitchell is thanked for TEM analysis and Dr. I. Czekaj for XPS measurements.

\section{Notes and references}

${ }_{80}{ }^{a}$ Institute for Chemical and Bioengineering, Department of Chemistry and Applied Biosciences, ETH Zurich, Wolfgang-Pauli-Strasse 10, CH8093 Zurich, Switzerland.

E-mails:jpr@chem.ethz.ch; cecilia.mondelli@chem.ethz.ch

Fax: +41 44 6331405; Tel: +41 446337120.

$85 \dagger$ Electronic Supplementary Information (ESI) available: XRD patterns and $\mathrm{N}_{2}$ isotherms of Sn-MFI, Ti-MFI, CBV720 and CVB720-ATGa, UVVis spectrum of Ti-MFI, and characterisation data of the USY zeolites used in this study. See DOI: 10.1039/b000000x/

1 (a) P. N. R. Vennestrøm, C. M. Osmundsen, C. H. Christensen, E. 90 Taarning, Angew. Chem., Int. Ed., 2011, 50, 10502. (b) P. Y. Dapsens, C. Mondelli, J. Pérez-Ramírez, ACS Catal., 2012, 2, 1487. 
2 H. Seyednejad, A. H. Ghassemi, C. F. van Nostrum, T. Vermonden, W. E. J. Hennink, Controlled Release, 2011, 152, 168.

3 M. Dusselier, P. Van Wouwe, A. Dewaele, E. Makshina, B. F. Sels, Energy Environ. Sci., 2013, 6, 1415.

${ }_{5} 4$ Available at: http://www.transparencymarketresearch.com/glycolicacid-market.html\#sthash.x0g3VLpI.dpuf, retrieved September 2013.

5 D. J. Loder, US Patent 2152852, 1939

6 (a) R. DiCosimo, A. Panova, D. P. O'Keefe, J. Thompson, M. S. Payne, US Patent 7473545, 2009. (b) F. Ebmeyer, H. Haberlein, H.

10 H. Haberlein, J. T. Haberlein, M. C. Haberlein, H. Mohn, US Patent 5723662. (c) B. Scharbert, P. Babusiaux, US Patent 5395488.

7 J. Zhang, X. Liu, M. Sun, X. Ma, Y. Han, ACS Catal., 2012, 2, 1698.

8 (a) P. Maki-Arvela, T. Salmi, B. Holmbom, S. Willfor, D. Yu Murzin, Chem Rev., 2011, 111, 5638. (b) D. K. Mishra, A. A.

15 Dabbawala, J. S. Hwang, J. Mol. Catal., A, 2013, 376, 63. (c) M. Yadav, D. K. Mishra, J. S. Hwang, Appl. Catal., A, 2012, 425, 110. (d) Z. Huang, F. Li, B. Chen, F. Xue, Y. Yuan, G. Chen, G. Yuan, Green Chem., 2011, 13, 3414. (e) J. Sun, H. Liu, Green Chem., 2011, $\mathbf{1 3}, 135$.

209 (a) M. Ziolek, I.Sobczak, P. Decyk, L. Wolski, Catal. Commun., 2013, 37, 85. (b) A. Takagaki, A. Tsuji, S. Nishimura, K. Ebitani, Chem. Lett., 2011, 40, 150. (c) M. Sankar, N. Dimitratos, D. W. Knight, A. F. Carley, R. Tiruvalam, C. J. Kiely, D. Thomas, G. J. Hutchings, ChemSusChem, 2009, 2, 1145.

2510 (a) T. A. Geissman, Org. React., 1944, 2, 94. (b) H. Wittcoff, Org. Synth., 1963, 4, 907. (c) R. M. Kellogg, in Comprehensive Organic Synthesis, ed. B. M. Trost and I. Fleming, Pergamon Press, Oxford, 1991, vol. 8.

11 D. Mohan ,C. U. Pittman, Jr., P. H. Steele, Energy Fuels, 2006, 20, 848 .

12 M. Balat, M. Balat, E. Kirtay, H. Balat, Energy Convers. Manage,, 2009, 50, 3147.

13 Available at: http://www.biofuelstp.eu/bio-oil.html, retrieved in September 2013.

3514 K. Sipilä, E. Kuoppala, L. Fagernäs, A. Oasmaa, Biomass Bioenergy 1998, 14, 103.

15 P. Van Wouwe, M. Dusselier, A. Basic, B. F. Sels, Green Chem., 2013,15, 2817.

16 T. Ohshima, Y. Yamamoto, U. Takaki, Y. Inoue, T. Saeki, K. Itou,

40 Y. Maegawa, T. Iwasakia, K. Mashima, Chem. Commun., 2009, 2688.

17 (a) E. Taarning, S. Saravanamurugan, M. S. Holm, J. Xiong, R. M. West, C. H. Christensen, ChemSusChem, 2009, 2, 625. (b) Y. Román-Leshkov, M. Moliner, J. A. Labinger, M. E. Davis, Angew.

45 Chem., Int. Ed., 2010, 49, 8954. (c) V. Choudhary, A. B. Pinar, S. I. Sandler, D. G. Vlachos, R. F. Lobo, ACS Catal., 2011, 1, 1724. (d) M. Moliner, Dalton Trans., 2013, DOI: 10.1039/C3DT52293H.

18 (a) M. S. Holm, S. Saravanamurugan, E. Taarning, Science, 2010, 328, 602. (b) F. de Clippel, M. Dusselier, R. Van Rompaey, P. Vanelderen, J. Dijkmans, E. Makshina, L. Giebeler, S. Oswald, G. V. Baron, J. F. M. Denayer, P. P. Pescarmona, P. A. Jacobs, B. F. Sels, J. Am. Chem. Soc., 2012, 134, 10089. (c) L. Li, C. Stroobants, K. Lin, P. A. Jacobs, B. F.Sels, P. P. Pescarmona, Green Chem., 2011, 13, 1175. (d) Q. Guo, F. Fan, E. A. Pidko, W. N. P. van der Graaff, Z. Feng, C. Li, E. J. M. Hensen, ChemSusChem, 2013, 6, 1352.

19 (a) P. Y. Dapsens, C. Mondelli, J. Pérez-Ramírez, ChemSusChem, 2013, 6, 831. (b) P. Y. Dapsens, M. J. Menart, C. Mondelli, J. PérezRamírez, Green Chem., DOI: 10.1039/C3GC40766G. (c) P. Y. Dapsens, B. T. Kusema, C. Mondelli, J. Pérez-Ramírez, J. Mol. Catal., A, 2013, DOI: 10.1016/j.molcata.2013.09.032.

20 K. Miltenberger, in Ullmann's Encyclopedia of Industrial Chemistry, VCH: Weinheim, 2000, vol. 18, pp. 481-492.

21 D. -G. Huang, X. Zhang, B. -H. Chen, Z. -S. Chao, Catal. Today, 2010, 158, 510.

6522 N. K. Mal, V. Ramaswamy, P. R. Rajamohanan, A.V. Ramaswamy, Microporous Mater., 1997, 12, 331.

23 M. R. Bendall, D. M. Doddrell, D. T. Pegg, J. Am. Chem. Soc., 1981, $\mathbf{1 0 3}, 4603$.

24 A. J. Shaka, J. Keeler, T. Frenkiel, R. Freeman, J. Magn. Reson., 70 $1983, \mathbf{5 2}, 335$.
25 C Rae, S. J. Berners-Price, B. T. Bulliman, P. W. Kuchel, Eur. J. Biochem., 1990, 193, 83.

26 P. P. Pescarmona, K. P. F. Janssen, C. Delaet, C. Stroobants, K. Houthoofd, A. Philippaerts, C. De Jonghe, J. S. Paul, P. A. Jacobs, B. F. Sels, Green Chem., 2010, 12, 1083.

27 C. M. Lew, N. Rajabbeigi, M. Tsapatsis, Microporous Mesoporous Mater., 2012, 153, 55.

28 C. M. Osmundsen, M. S. Holm, S. Dahl, E. Taarning, Proc. R. Soc. A 2012, 468, 2000.

8029 (a) C. Hammond, S. Conrad, I. Hermans, Angew. Chem., Int. Ed., 2012, 51, 11736 (b) J. Dijkmans, D. Gabriëls, M. Dusselier, F. de Clippel, P. Vanelderen, K. Houthoofd, A. Malfliet, Y. Pontikes, B. F. Sels, Green Chem., 2013, 15, 2777.

30 J. Pérez-Ramírez, S. Mitchell, D. Verboekend, M. Milina, N.-L.

85 Michels, F. Krumeich, N. Marti, M. Erdmann, ChemCatChem, 2011, 3, 1731.

31 A. Corma, L. T. Nemeth, M. Renz, S. Valencia, Nature, 2001, 412, 423.

32 M. Boronat, P. Concepción, A. Corma, M. Renz, S. Valencia, J Catal., 2005, 234, 111.

33 R. Bermejo-Deval, R. Gounder, M. E. Davis, ACS Catal., 2012, 2, 2705 .

34 S. Roy, K. Bakhmutsky, E. Mahmoud, R. F. Lobo, R. J. Gorte, ACS Catal., 2013, 3, 573.

9535 Y. K. Hwang, T. H. Jin, J. M. Kim, Y.-U. Kwon, S.-E. Park, J.-S. Chang, J. Nanosci. Nanotechnol., 2006, 6, 1.

36 H. Y. Luo, L. Bui, W. R. Gunther, E. Min, Y. Román-Leshkov, ACS Catal., 2012, 2, 2695.

37 R. Bermejo-Deval, R. S. Assary, E. Nikolla, M. Moliner, Y. Román100 Leshkov, S. Hwang, A. Palsdottir, D. Silverman, R. F. Lobo, L. A. Curtiss, M. E. Davis, Proc. Natl. Acad. Sci. U. S. A., 2012, 109, 9727.

38 G. Yu, A. R. Bayer, M. M. Galloway, K. J. Korshavn, C. G. Fry, F. N. Keutsch, Environ. Sci. Technol., 2011, 45, 6336. 


\section{Electronic Supplementary Information}

\section{Continuous Process for Glyoxal Valorisation using Tailored Lewis-acid Zeolite Catalysts}

Pierre Y. Dapsens, Cecilia Mondelli*, Bright T. Kusema, René Verel and Javier Pérez-Ramírez*

Institute for Chemical and Bioengineering, Department of Chemistry and Applied Biosciences, ETH Zurich, Wolfgang-Pauli-Strasse 10, CH-8093 Zurich, Switzerland. Fax: +41 446331405 Tel: +41 446337120 ; E-mails: cecilia.mondelli@chem.ethz.ch; jpr@chem.ethz.ch.
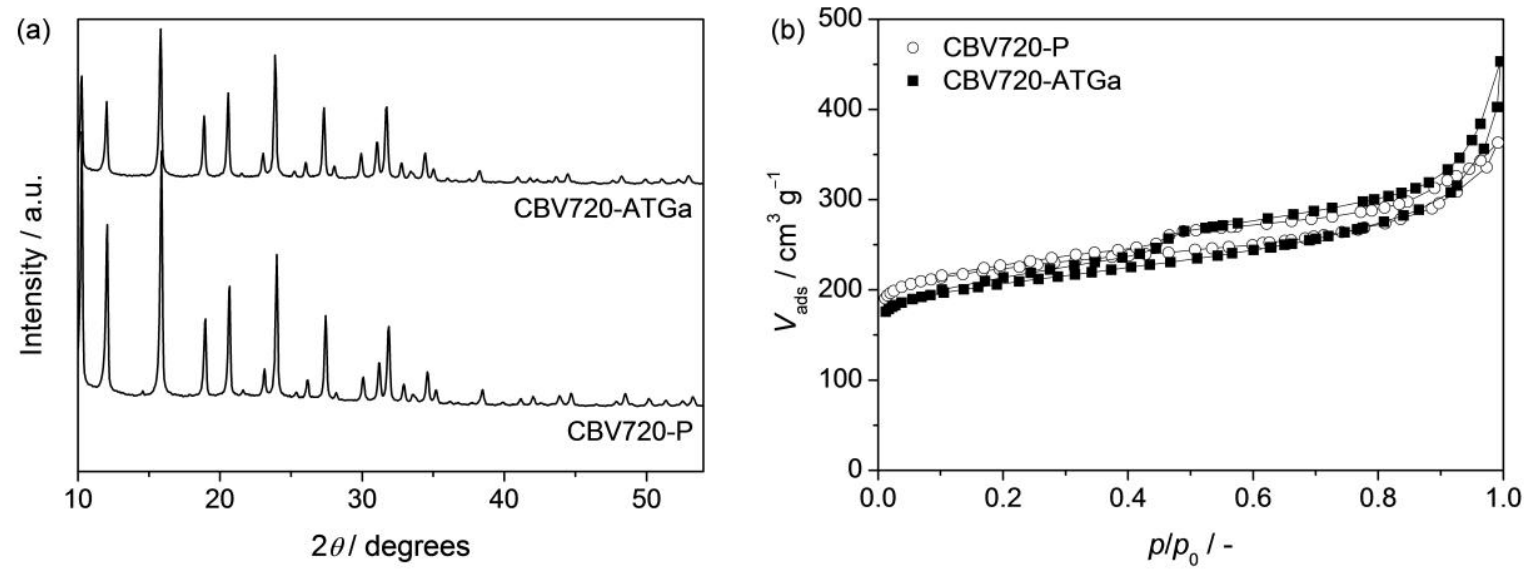

Fig. SI1. (a) X-ray diffraction patterns and (b) $\mathrm{N}_{2}$ isotherms of CBV720 in parent form (P) and after alkaline-assisted galliation (ATGa).

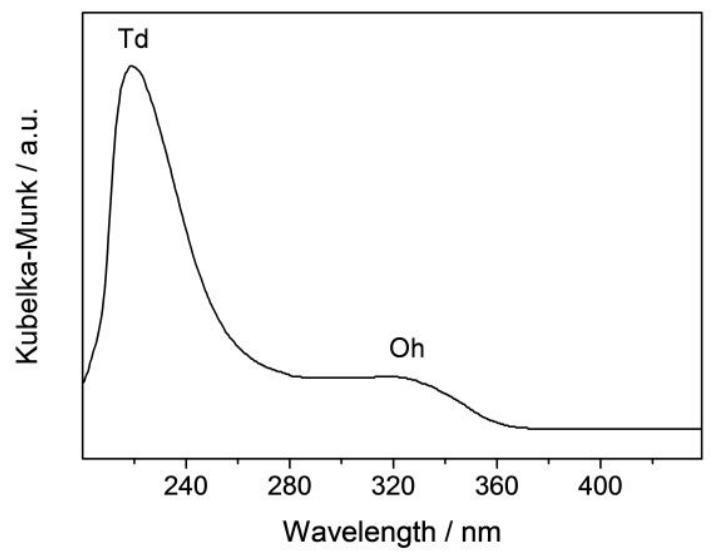

Fig. SI2. Diffuse reflectance UV-Vis spectrum of Ti-MFI. The bands can be assigned to tetrahedral (Td) titanium in lattice positions of the zeolite and octahedral (Oh) titanium in extra-framework positions. 

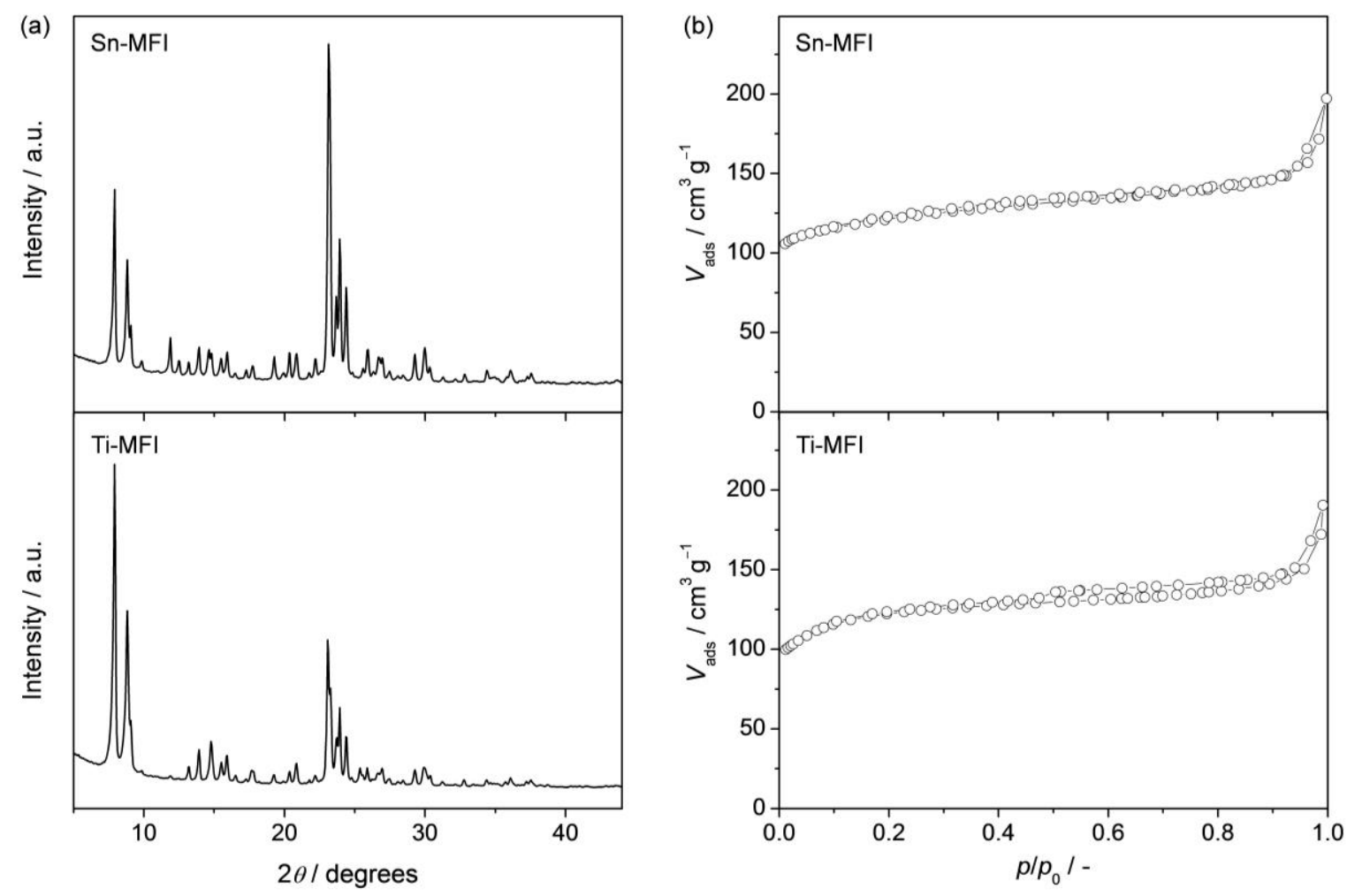

Fig. SI3. (a) X-ray diffraction patterns and (b) $\mathrm{N}_{2}$ isotherms of Sn-MFI and Ti-MFI.

Table SI1 Characterisation data of the USY zeolites

\begin{tabular}{lccccc}
\hline Catalyst & $\begin{array}{c}\mathrm{Si} / \mathrm{Al}^{a} \\
\left(\mathrm{~mol} \mathrm{~mol}^{-1}\right)\end{array}$ & $\begin{array}{c}V_{\text {pore }}^{b} \\
\left(\mathrm{~cm}^{3} \mathrm{~g}^{-1}\right)\end{array}$ & $\begin{array}{c}V_{\text {micro }^{c}} \\
\left(\mathrm{~cm}^{3} \mathrm{~g}^{-1}\right)\end{array}$ & $\begin{array}{c}S_{\text {meso }}{ }^{c} \\
\left(\mathrm{~m}^{2} \mathrm{~g}^{-1}\right)\end{array}$ & $\begin{array}{c}S_{\mathrm{BET}^{d}} \\
\left(\mathrm{~m}^{2} \mathrm{~g}^{-1}\right)\end{array}$ \\
\hline CBV300 & 2.55 & 0.37 & 0.33 & 34 & 843 \\
CBV500 & 2.6 & 0.36 & 0.24 & 83 & 675 \\
CBV600 & 2.6 & 0.39 & 0.23 & 76 & 651 \\
CBV712 & 6.0 & 0.48 & 0.28 & 113 & 807 \\
CBV720 & 17 & 0.56 & 0.29 & 128 & 842 \\
CBV760 & 30 & 0.56 & 0.31 & 149 & 903 \\
CBV780 & 40 & 0.54 & 0.30 & 139 & 859 \\
HSZ390-HUA & 250 & 0.57 & 0.29 & 116 & 825
\end{tabular}

${ }^{a}$ According to the manufacturer's specifications. ${ }^{b}$ Volume adsorbed at $p / p_{0}=0.99 .{ }^{c}$ Determined by the $t$-plot method. ${ }^{d}$ Determined by the BET method. 